\title{
A Case Report of an Adult-onset Niemann-Pick Disease Type C Presenting as Bipolar-affective Disorder
}

\author{
${ }^{1}$ Kirti Sharma, ${ }^{2}$ Ravi Sharma
}

\section{ABSTRACT}

Aim: We are presenting a case report of an adult-onset NiemannPick disease type C (NP-C) presenting as a bipolar-affective disorder.

Background: The NP-C is a rare autosomal recessive lysosomal storage disorder. Clinical presentations of NP-C feature a range of systemic and neurological signs that arise at different ages and progress at different rates. Many of the neurological and psychiatric signs of the disease are shared with other neurological conditions, and NP-C is therefore often overlooked.

Case Description: A 43-year male was under psychiatric treatment for the last 20 years without any improvement in the diagnosis of "bipolar-affective disorder." Presence of vertical gaze palsy with cerebellar ataxia, extrapyramidal symptoms and cognitive decline over a long progressive course of illness made Niemann-Pick (NP) disease as a possible diagnosis. The diagnosis was confirmed by low sphingomyelinase levels.

Conclusion: Early diagnosis of the disease is helpful in providing appropriate psychiatric and neurological management along with genetic counseling. To ensure rapid diagnosis and proper management, it is important that clinicians are familiar with this obscure condition.

Clinical significance: The gradual progression of NP-C poses a diagnostic challenge. This patient was managed for psychiatric illness without improvement. The gradual appearance and progression of neurological symptoms was either masked by the behavioral disturbances or was attributed to the use of psychotropic medication. The psychiatric manifestations can overshadow the subtle neurological signs of NP-C, which leads to significant delays in the detection and diagnosis of NP-C.

Keywords: Bipolar-affective disorder, Niemann-Pick disease type C (NP-C), Vertical gaze palsy.

How to cite this article: Sharma K, Sharma R. A Case Report of an Adult-onset Niemann-Pick Disease Type C presenting as Bipolar-affective Disorder. Journal of Postgraduate Medicine, Education and Research, Oct-Dec 2018;52(4):177-179.

\section{Source of support: Nil}

Conflict of interest: Nil

\footnotetext{
${ }^{1}$ Junior Resident, ${ }^{2}$ Professor and Head

1,2Department of Psychiatry, Indira Gandhi Medical College Shimla, Himachal Pradesh, India
}

Corresponding Author: Ravi Sharma, Professor and Head Department of Psychiatry, Indira Gandhi Medical College, Shimla, Himachal Pradesh, India, e-mail: dr.ravicsharma@gmail.com

\section{CASE REPORT}

\section{Background}

The NP-C is a rare autosomal recessive lysosomal storage disorder. The adult onset of NP disease is caused by mutations in the NPC1 gene (in $95 \%$ of cases) or the NPC2 gene. Clinical presentations of NP-C feature a range of systemic and neurological signs that are not specific to the disease, arising at different ages, and progressing at different rates. They are also shared with other conditions that involve progressive neurological deterioration, and NP-C is often overlooked. ${ }^{1}$

\section{CASE DESCRIPTION}

A 43-year male diagnosed case of bipolar-affective disorder was brought with 20 years of progressive psychiatric illness without any improvement. His birth and developmental history were within normal limits. He had received formal high school education. The illness started 20 years ago with behavioral changes in the form of suspiciousness towards colleagues, aggression, increased activity, grandiose ideas, muttering and smiling to self, social withdrawal and poor self-care. The patient was treated with antipsychotics and modified electro-convulsive therapy (ECT). Two years later he had a depressive episode with the delusion of persecution, the delusion of infidelity and third-person auditory hallucinations similar to the first episode. There was some improvement in depressive symptoms; however, the psychotic symptoms persisted. A year later he had a manic episode with persisting psychotic symptoms. For the next 10 years, the patient continued to have similar episodes of mania and depression. He had incomplete remission despite being on mood stabilizers and antipsychotics in different combinations. He did not respond to an adequate trial of divalproex, lithium, risperidone, olanzapine, quetiapine, and clozapine. He frequently developed extrapyramidal side effects to psychotropic medication and was unable to tolerate them even at low doses. The patient also began exhibiting signs and symptoms suggestive of a neurological disorder such as an inability to maintain posture, tendency to fall back while sitting and sustaining injuries after frequent falls while walking, fecal and urinary urge incontinence along with social disinhibition. He also developed rigidity in his upper and lower limbs which would aggravate on the administration of antipsychotics. 
At the time of presentation, the patient was highly suspicious, demanding frequent sex, having an unduly cheerful mood, decreased sleep, slurring of speech, unstable gait and urinary urge incontinence. There was no family history of any psychiatric or neurological illness. General physical and systemic examination was unremarkable but for neurological examination which revealed vertical gaze palsy, horizontal nystagmus, dysarthria, increased muscle tone, brisk deep tendon reflexes of all four limbs and impaired cerebellar signs. His score on mini-mental state examination (MMSE) was 21/30. Mental status examination revealed slurred speech, euphoric effect, grandiose ideas, the delusion of infidelity, cognitive impairment and impaired insight.

All routine blood investigations along with chest X-ray and ultrasonography abdomen were within normal limits. Magnetic resonance imaging (MRI) brain revealed patchy and confluent $\mathrm{T} 2$ signal hyperintensities in centrum semiovale and periventricular white matter suggestive of chronic microangiopathy (Fig. 1). Sphingomyelinase activity was deficient $(<0.5)$, bone marrow aspiration could not be done as the patient denied consent for this invasive test while facility for genetic testing was costly and not available in our State.

The patient was discharged after 6 weeks of hospital stay on haloperidol, $5 \mathrm{mg}$ /day, quetiapine $400 \mathrm{mg} /$ day, carbamazepine $600 \mathrm{mg} /$ day, trihexyphenidyl $2 \mathrm{mg} /$ day and tetrabenazine $75 \mathrm{mg} /$ day. There was a marked improvement in behavioral symptoms of suspiciousness and hypersexuality and some improvement in tremors and ataxia.

\section{DISCUSSION}

This patient presented with 20 years of neuropsychiatric illness. The first 10 years of the course of illness were

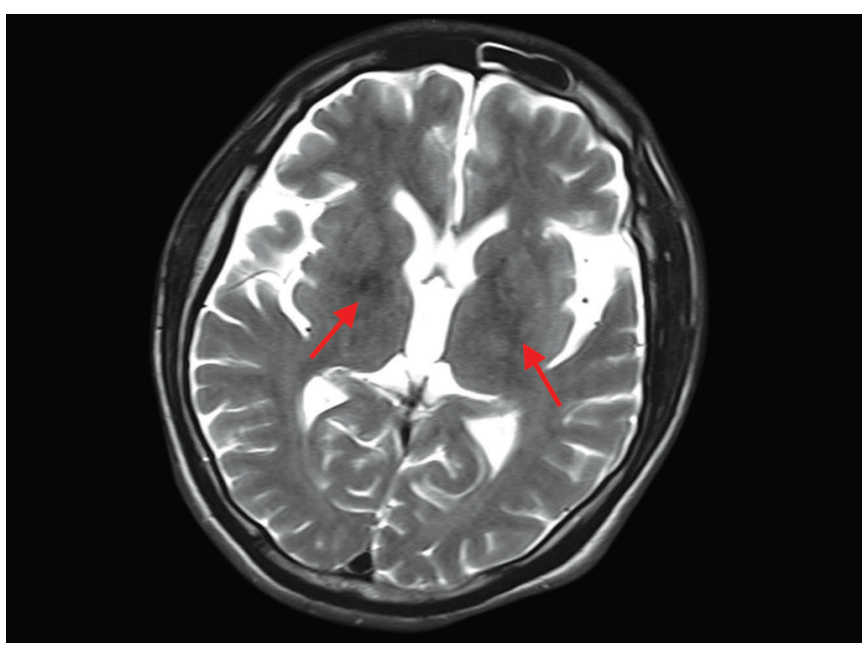

Fig. 1: MRI T2-weighted image showing hyperintensities in periventricular white matter (Red Arrows) characterized by psychiatric symptoms after which neurological symptoms emerged. They were initially either masked by the behavioral disturbances or were attributed to the use of psychotropic medication. Longitudinal history and examination helped us consider organic (neurologic) etiology for two reasons. Firstly, in spite of continued treatment with psychotropics, illness had a progressive, deteriorating course with no response to any medication. Secondly, the patient was unable to tolerate psychotropics even at sub-therapeutic doses. Chronic ischaemic changes seen on MRI brain along with neurological examination raised the possibility of an underlying metabolic disorder. Presence of vertical gaze palsy, cerebellar ataxia, extrapyramidal symptoms and cognitive decline over a long progressive course of illness made NP-C disease as a possible diagnosis. The diagnosis was confirmed by low sphingomyelinase levels.

Malhotra et al. have reported a similar case of NP-C disease, presenting as schizophrenia, diagnosed after 14 years of onset of neuropsychiatric illness. They recommended that the presence of soft neurological signs and symptoms in a case of psychosis should alert the possibility of an underlying medical illness. ${ }^{2}$ Another case of NP-C presenting as a bipolar disorder was reported by Sullivan et al. ${ }^{3}$ A case series of 13 patients diagnosed with adult-onset NP-C from France revealed that the first presenting signs in adulthood were psychiatric problems (38\%), cognitive difficulties (23\%), ataxia $(20 \%)$, movement disorders such as dystonia $(11 \%)$, vertical gaze palsy $(8 \%)$, dysarthria $(8 \%)$ and epilepsy $(3 \%)$. First neurological symptoms occurred within the second or third decades in most patients, all patients followed normal schooling, and most of them acquired professional training or had sought employment, meaning that their neurological involvement was not significant until adulthood. ${ }^{4}$ The same trend was observed in our patient as well. An ongoing international, prospective observational cohort study in the form of the NP-C Registry has 39 patients of adult onset of the disease out of 163 patients enrolled in the study. Sixteen of these 39 patients had 'psychiatric' manifestations at the time of enrolment with a long lag time between the mean age at neurological onset and age at diagnosis. ${ }^{5} \mathrm{NP}-\mathrm{C}$ can express as an isolated psychiatric-disorder, such as schizophrenialike psychosis, depression, bipolar disorder, obsessivecompulsive behaviour and behavioural problems (sleep disorders, hyperactivity, agitation, aggressiveness or self-mutilation). These psychiatric manifestations are mostly atypical and usually accompanied by visual hallucinations, confusion, symptom fluctuations, progressive cognitive decline, treatment resistance or aggravation with neuroleptic drugs. ${ }^{6}$ 


\section{CONCLUSION}

The NP-C frequently presents with atypical psychiatric manifestations areaccompanied by visual hallucinations, confusion, symptom fluctuations, progressive cognitive decline, treatment resistance or aggravation with neuroleptic drugs. The late appearance of neurological manifestations is often wrongfully attributed to the effects of antipsychotic medication, which generates a tardy diagnosis.

As in our patient, psychiatric manifestations can overshadow the subtle neurological signs of NP-C, which leads to significant delays in the detection and diagnosis of NP-C.

\section{CLINICAL SIGNIFICANCE}

The NP-C is particularly difficult to diagnose when presenting as a real psychiatric disorder, frontal dementia, ataxia or dystonia. Early diagnosis of the disease is helpful in providing appropriate psychiatric and neurological management along with genetic counseling. Treatment should be tailored to the needs of the patient to provide symptomatic relief and decrease the caregiver burden.

\section{REFERENCES}

1. Vanier MT. Niemann-Pick disease type C. Orphanet Journal of Rare Diseases. June 2010;5(1):16.

2. Malhotra HK, Isenberg NB, Barash L. Niemann-Pick Disease Type $C$ presenting as schizophrenia. Primary Psychiatry, August 2003;10(8):74-76.

3. Sullivan D, Walterfang M, Velakoulis D. Bipolar disorder and Niemann-Pick disease type C. American Journal of Psychiatry. 2005 May 1;162(5):1021-1022.

4. Sévin M, Lesca G, Baumann N, Millat G, Lyon-Caen O, Vanier MT, et al. The adult form of Niemann-Pick disease type C. Brain. 2006 Sep 26;130(1):120-133.

5. Patterson MC, Mengel E, Wijburg FA, Muller A, Schwierin B, Drevon $\mathrm{H}$, et al. Disease and patient characteristics in NP-C patients: findings from an international disease registry. Orphanet journal of rare diseases. 2013 Dec;8(1):12.

6. Shulman L M, David N J, Weiner W J. Psychosis as the initial manifestation of adult-onset Niemann-Pick disease type C. Neurology, September 1995;45(9):1739-1743. 